\title{
The Research on the Sports Tourism Resources Development
}

\section{Evaluation}

\author{
Ren Yadong \\ Huaiyin Normal University, Jiangsu, Huaian223300 \\ 64327809@163.com
}

Keywords: Sports tourism resources evaluation; Analytic hierarchy process; Tibet

\begin{abstract}
With the improvement of people's income and living standard, people's spiritual life has also been constantly enriched, and the consumption concept has changed consequently. People have more and more intense desire and demand on the tourism after the intense work. While as the emerging mode of traveling, the sports tourism is loved by people for its produced profound experience and uniqueness to the tourists. Carrying out the right development and reasonable utilization on the sports tourism in Tibet can promote the development of sports tourism in Tibet, and at the same time drive the development of other industrial chain so as to produce the positive effect on the sustainable development of Tibet and even the entire western areas.
\end{abstract}

\section{Introduction}

The sports tourism mainly takes the outdoor sports as the main subject, and takes the natural environment as the development carrier. And Tibet has the obvious advantages both on the natural environment and human geography. The sports tourism resources in Tibet are extremely abundant. No matter what it is, the high mountain and snow summit resources, the river and valley resources, or the lake resources, all of them show their great development potentials. In addition, the region's ethnic sports culture has its distinct characteristics, and all of the things above can be the favorable conditions for the development of sports tourism resources in Tibet.

Relevant literatures of existing sports tourism indicate that there are many countermeasure researches of existing sports tourism resource development, but there are few countermeasure researches based on the development of sports tourism resources evaluation. There are qualitative evaluation researches in the sports tourism resources evaluation researches, and there are few scientific, comprehensive and quantitative evaluation researches. The systematic research on the sports tourism resources evaluation theory is less involved. Based on the previous research, we use the method of expert interview and analytic hierarchy process to establish the sports tourism resources evaluation system so as to enrich and perfect the sports tourism resources evaluation theory in this paper.

\section{The Construction of Sports Tourism Resources Evaluation System}

The Construction Principles of Sports Tourism Resources Evaluation Indicator. Representativeness. The selection and confirmation of the sports tourism resources evaluation indictor should have representativeness and typicality. They can fully reflect the value, the natural properties, the development condition and the development value characteristics of sports tourism resources. 
Completeness. The sports tourism resources evaluation indictor should give the full consideration to the requirement of completeness. As far as the evaluation objects are concerned, we not only need to consider the natural sports tourism resources value, but also need to consider the sports tourism value with Chinese characteristics. As far as the category evaluation is concerned, we not only need to consider the sports tourism resources value evaluation, but also need to consider its development value and development conditions.

Scientificity. The indictor system should be able to accurately and objectively reflect the hierarchy and the value of the sports tourism resources. Therefore, in the process of formulating the indictor system we should give the continuous research and analysis, and try to adjust and optimize so as to avoid the simple pile of statistical indicators and the repetition between the indictors. We should choose the indicator which can best reflect the important factors of sports tourism resources evaluation as far as possible.

Operability. For the setting of evaluation indictor we should consider its simplification and its easy implement to avoid using the indictor which is hard to obtain and measure so as to make the evaluation have the operability and ensure the feasible evaluation process.

The Construction of Sports Tourism Resources Evaluation System. For the first step, we should combine with the precious existing literature researches and take them as the basis to form the basic framework of Sports tourism resources evaluation indictor system.

For the second step, we should carry out a comprehensive and thorough investigation based on this object, namely sports tourism resources in Tibet to fully refer the characteristics of the local sports tourism resources and revise the prototype of the indictor system.

For the third step, we should consult with 31 experts respectively in the subject areas of geography circles, the tourism circles, the sports circles of Tibet University, Lhasa Teachers College, Tibet Agriculture and Animal Husbandry College, Tibet Sports Technical School, Tibet Mountaineering School. And then we combine with the experts' opinions to perfect the evaluation indictor system. And eventually the evaluation indictor system is confirmed as follows. It includes 3 first class indicators, 11 second class indicators and 22 third class indicators.

The Weight Confirmation of Sports Tourism Resources Evaluation Indictor. The Confirmation Steps of the Indictor Weight. We should construct the judgment matrixes.Assume that $\mathrm{A}$ is the corresponding upper hierarchy indictor of a1, a2 $\cdots$ an, then aij refers to a judgment of the relative importance of aj to $\mathrm{A}$ and ai.

We should make aij (i, $\mathrm{j}=1,2,3, \ldots, \mathrm{n})$ generally take the values as $1,2,3,4,5,6,7,8,9$ and so on, the 9 rank scales. Their specific meanings are shown in the Table 1.

Table 1 "the 1-9 scale" and its meaning

\begin{tabular}{l}
\hline $\begin{array}{l}\text { The Scale with } \\
\text { the Importance }\end{array}$ \\
\hline
\end{tabular}

1 Compared with the two factors, and both of them have the same importance.

3 Compared with the two factors, and one of them is more important slightly.

$5 \quad$ Compared with the two factors, and one of them is more important obviously.

$7 \quad$ Compared with the two factors, and one of them is more important intensively.

9 Compared with the two factors, and one of them is more important extremely.

2, 4, 6, $8 \quad$ The medium value of the above adjacent evaluation can be described as follows.

1) important slightly; 2) more important; 3) very important; 4) more important 
intensively.

Reciprocal Compared with the two large indictors, ai and aj, we obtain the judgment aij. From

this we know that we compare ai and aj to obtain the judgment aji=1/aij.

Generally speaking, the numerical value of judgment matrix is confirmed jointly by three aspects. First, we should refer to the relevant data of research object. Next, we should refer to the industry experts and scholars' advices and opinions. Finally, we should synthesize the understanding of the researcher himself about the problem. Assume that the judgment matrix shows the following relationship.

$$
j=\frac{\text { aik }}{\text { ajk }} \quad(\mathrm{I}, \mathrm{j}, \mathrm{k}=1,2,3, \cdots, \mathrm{n})
$$

Then, we think that the judgment matrix has the so-called complete consistency. However, because the objective things have bigger complexity, along with different people have the differentiated cognitions, the deviation is inevitable. Therefore, it is not realistic to stipulate that all the judgment matrixes show the complete conformity, especially in the situation with more influencing factors. So in order to evaluate the scientific nature and accuracy of the results with hierarchical analysis process, we need to take the judgment matrix as the object and make the strict inspection on its consistency.

The Hierarchical Single Arrangement

As far as the hierarchical single arrangement is concerned, its fundamental purpose is to be based on the corresponding element in the upper hierarchy to achieve the corresponding weight value associated with it within the hierarchy.

As far as the hierarchical single arrangement is concerned, its essence is to be based on the corresponding characteristic roots and character vectors to do the corresponding calculations. Specially speaking, according to the judgment matrix A, we should adopt the following computational formula.

$\mathrm{AW}=\lambda \max \mathrm{W}$

In the formula above, $\lambda$ max refers to the largest eigenvalue of $A$, and $W$ refers to the corresponding normalized eigenvector of $\lambda$ max. Thus, the component $\mathrm{Wi}$ of $\mathrm{W}$ is the corresponding weighted value of the corresponding indicator with the hierarchical single arrangement.

For the judgment matrix A, when it meets the condition of complete consistency, $\lambda$ max $=\mathrm{n}$. However, under the general case, this situation does not exist. In order to achieve the effective test of the corresponding consistency of judgment matrix, it is necessary to calculate accordingly aiming at the consistency indictor.

$$
C I=\frac{\lambda_{\max -n}}{n-1}
$$

When $\mathrm{CI}=0$, then it shows that the judgment matrix meets the requirements of the complete consistency. On the contrary, with the continuous increase of CI, the corresponding consistency of judgment matrix will get worse.

When the random consistency is less than 0.10, we think that the judgment matrix has the ideal consistency. When $\mathrm{CR}$ is larger than or equal to 0.10 , we should adjust the judgment matrix accordingly till its consistency meets the relevant requirements.

The Hierarchy General Ranking

When we know that the corresponding single arrangement result of all hierarchies at a certain hierarchy, we can confirm the corresponding weight value of the importance for all the elements of 
this hierarchy with the upper hierarchy as the relative object by calculation. This is so-called hierarchy general ranking. In the process of hierarchy general ranking, we should carry out it based on "top-down hierarchy" order one by one. For the hierarchy general ranking, we also need to make the tightened inspection according to its consistency.

The Confirmation of Each Indictor Weight. The Adjustment of Matrix Data Acquisition. First, according to the correlations which have been confirmed between the hierarchical structures of each evaluation indictor we set up the survey form of the relative importance with the pair-wise adjustment about the indictors in the same hierarchy. 31 experts in the subject areas of geography circles, the tourism circles, the sports circles of Tibet University, Lhasa Teachers College, Tibet Agriculture and Animal Husbandry College, Tibet Sports Technical School, Tibet Mountaineering School fill out the survey form in this paper. We issue 31 questionnaires in total, including 29 valid questionnaires. Finally we deal with the survey forms respectively to construct the judgment matrix table of the corresponding experts. And we check to judge the consistency of the judgment matrix. For the judgment matrixes without passing the consistency check, we should return the questionnaires to the experts so as to adjust them till they pass the consistency check. That is to say, for the weights of the corresponding evaluation factors we can obtain the weight of each evaluation indictor in the general objective hierarchy through the hierarchy general ranking.

Finally the judgment matrix of each evaluation indictor CR is less than 0.10 . So we judge that the consistency of matrix is comparatively good and it conforms to the requirements of the consistency check to make the determined value of weights be effective.

Table 2 The Weight Table of Tibetan Sports Tourism Resources Evaluation Indictor (The Hierarchy General Ranking)

\begin{tabular}{|c|c|c|c|c|}
\hline $\begin{array}{l}\text { The Evaluation } \\
\text { Comprehensive }\end{array}$ & Weight & $\begin{array}{l}\text { The Evaluation } \\
\text { Project Hierarchy }\end{array}$ & Weight & $\begin{array}{l}\text { The Evaluation Factor } \\
\text { Hierarchy D }\end{array}$ \\
\hline
\end{tabular}

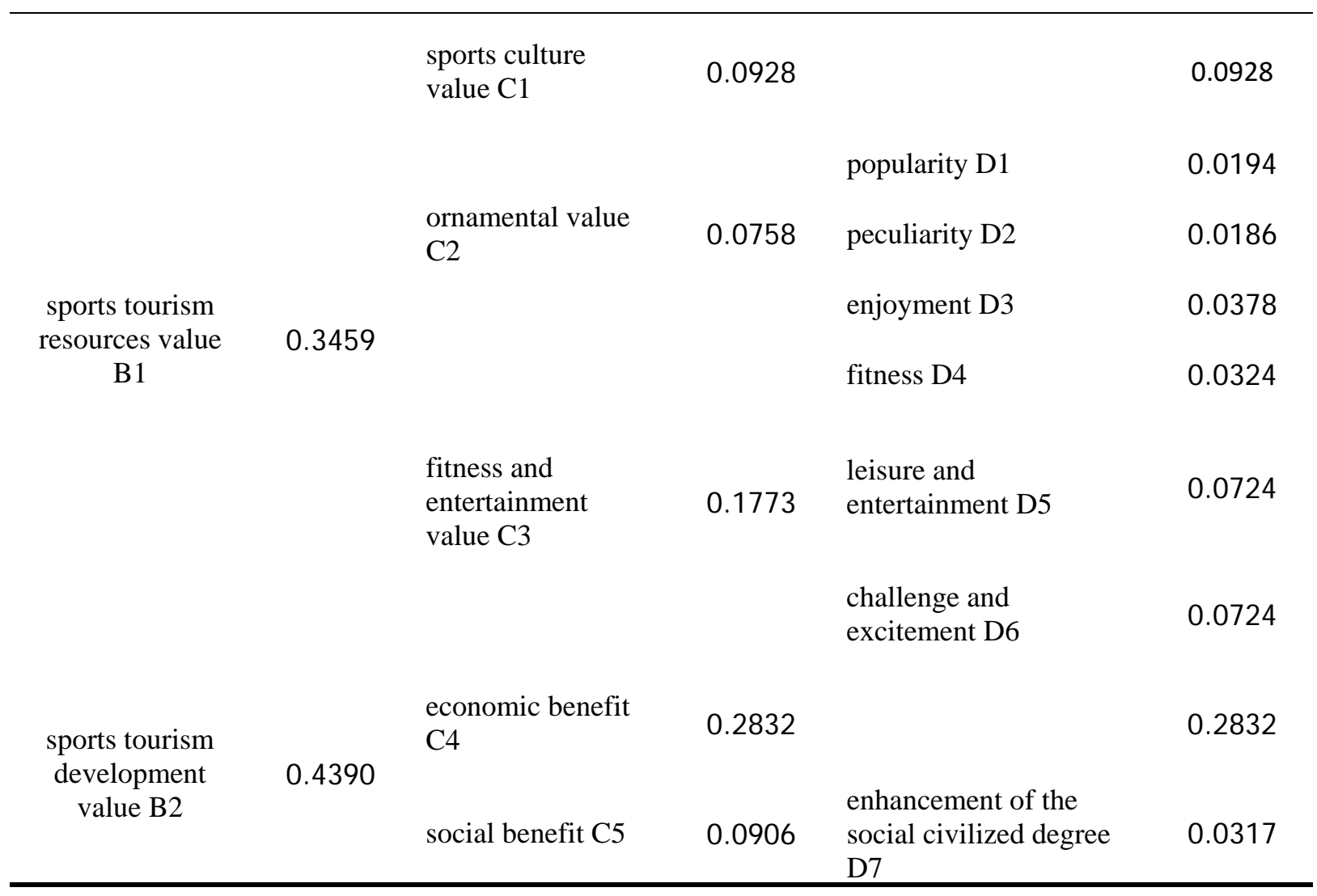




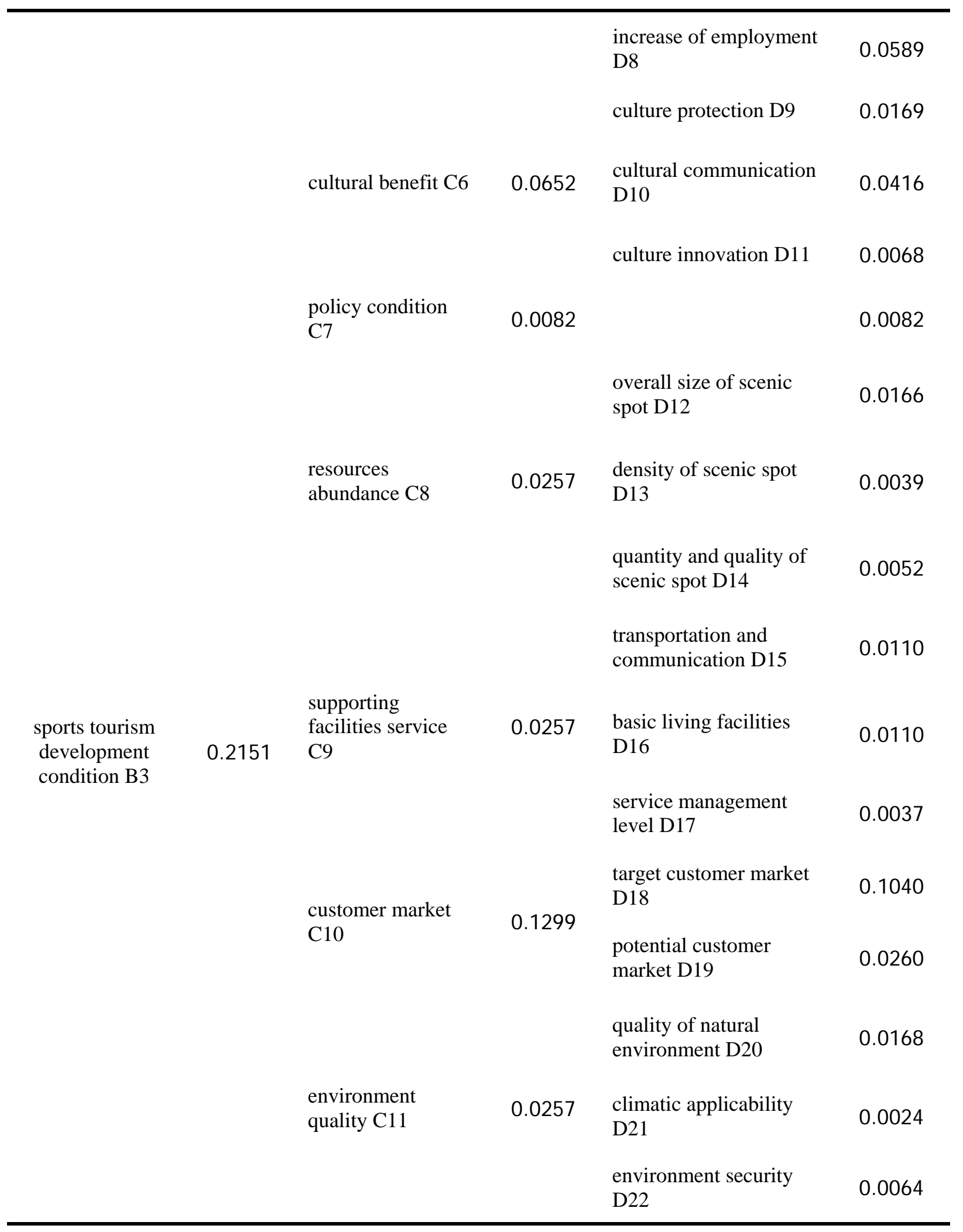

\section{The Analysis of Evaluation Results}

Known from the relevant data of table 2, the corresponding quantitative evaluation comprehensive value of the local sports tourism resources is 7.7788. According to the relevant classification standard, it should be included in the category of sports tourism resources with Level 4. The weight of sports tourism resources value is 0.3459 . The weight of development condition of sports tourism resources is 0.2151 . These illustrate that the sports tourism resources value and the 
development condition of sports tourism resources are the basis of achieving the development value. They are also one of the important factors which affect Tibetan sports tourism resources development. Seen from the setting score value, the sports culture value (the score value is 8.9501), and popularity of Tibetan sports tourism resources are both extremely high. And both of them have very strong challenge and excitement (the score value is 9.2337). The sports tourism resources have extremely high value of their own. At the same time the development of Tibetan sports tourism resources plays very important role in both of the protection (the score value is 8.0130) and communication (the score value is 9.1035) of Tibetan sports culture to help facilitate the inheritance and transmission of Tibetan sports culture. Seen from the policy condition (the score value is 9.2350), overall size of scenic spot (the score value is 8.1302), the quantity and quality of scenic spot (the score value is 9.2376), the target customer market (the score value is 9.1305), and the quality of natural environment (the score value is 9.3562), the Tibetan sports tourism resources have very high developable conditions. However, they have the relatively weak supporting facilities service. All of Tibetan transportation and communication (the score value is 4.1002 ), the basic living facilities (the score value is 4.3423) and the service management level (the score value is 4.683) can't meet the standards. These become the important reasons of hindering the Tibetan sports tourism resources to obtain the effective development. Compared with overall size of scenic spot (the score value is 8.1302), the quantity and quality of scenic spot (the score value is 9.2376), the score value of the density of scenic spot (the score value is 6.5617) is relatively low. It shows that the Tibetan sports tourism resources are extremely abundant. However, the area of Tibet is large, and the density of scenic spot is relatively weak. Therefore, we should strengthen the spatial layout and the integration efforts. We should make the combination and piece area division of Tibetan sports tourism scenic spot to make the planning as orderly line system so as to provide a good traffic environment for the development of local tourism.

In the evaluation hierarchy of sports tourism development condition, the weight of the customer market is the highest, and it is 0.1299 . It shows that the customer market is the key factor which affects the sports tourism development and the customer market affects the economic benefits of the sports tourism development so as to affect the development value of sports tourism resources. Its comprehensive score value is 1.1363 . And it has the highest proportion among the influence factors of development conditions. As the world-renowned tourist resort, every year many tourists at home and abroad come to visit Tibet with its famous name. What's more, it shows that the tendency increases greatly year by year, and it has very large tourist market space. The weight of policy condition is the lowest in the evaluation hierarchy and the score value is extremely high. It shows that the policy condition of Tibetan sports tourism resources development is very good, and the influence on the Tibetan sports tourism resources development is small relatively. The resources abundance, the supporting facilities service, and the environment quality are almost the same and their values are all 0.0257. However, the comprehensive score value of the supporting facilities service is only 0.1101. Compared with resources abundance (the comprehensive score value is 0.2083) and the environment quality (the comprehensive score value is 0.2063), it is extremely weak. It hints us that the corresponding supporting facilities system of the local sports tourism hasn't been established yet ideally so as not be able to meet the current needs and future development needs. If we want to develop the Tibetan sports tourism resources we need to promote the Tibetan supporting facilities service ability first and take the infrastructures as the object to make them get the organic integration. When we build and improve the transport network, at the same time we actively cultivate and output the high-level professional talents to promote the Tibetan traffic level, the communication level, the infrastructure level and the service management 
level so as to play a role in supporting the Tibetan sports tourism from the aspects of hardware and software.

\section{Reference}

[1] Flagestad, A. \& Hope C. A. Strategic Success in Winter Sports Destinations: A Sustainable Value Creation Perspective.Tourism Management, 2001, 22 (5): 445- 461.

[2] Simon Hudson. Sport and Adventure Tourism. The Haworth Hospitality Press, 2003.

[3] Antho ny, (1966). Sport and to ur ism. Londo n: Central Council fo r Physical Recreation.

[4] Standevan, (1998). Sport tourism: Joint marketingA starting point for beneficia synergies. Journal of Vacation Marketing, 4, 3951. 